\title{
The extreme synchronicity of stellar ages of red galaxies in the JKCS 041 cluster at $z=2.2$
}

\begin{abstract}
S. Andreon
Observatorio Astronomico di Brera, via Brera 28, 20121 Milan, Italy

e-mail: stefano.andreon@brera.inaf.it

Received 4 March 2011 / Accepted 22 March 2011

ABSTRACT

Above redshift $z \sim 1.4$, we known little or nothing about the stellar ages of red galaxies in clusters, yet at these high redshifts important changes are predicted by current renditions of galaxy formation models embedded in the standard hierarchical paradigm of structure formation. Red-sequence galaxies in the cluster JKCS 041 at $z=2.2$ show a tight distribution in colour that indicates a star formation history that is highly synchronized across galaxies. Specifically, we measure a spread in stellar age of $160 \pm 30 \mathrm{Myr}$, in marked disagreement with the current understanding of how massive red galaxies form in clusters, i.e. if they are produced somewhat stochastically in merging episodes that sometimes involve gas, hence star formation. The existence of a tight distribution in colour when the universe was at one quarter of its current age implies that mechanisms that have not yet been implemented in current galaxy formation scenarios long ago began to shape the star formation history of red cluster galaxies.
\end{abstract}

Key words. galaxies: evolution - galaxies: formation - galaxies: clusters: general - galaxies: clusters: individual JKCS 041

\section{Introduction}

In $\Lambda$ CDM cosmology there may be multiple epochs of galaxy formation depending on the phenomenon one concentrates on. Indeed, we expect that galaxies may be younger than their stars, because the mass is assembled hierarchically via the progressive merger of lower luminosity objects. The two epochs of star formation and mass assembly then may yield constraints on the mechanisms of galaxy formation and evolution. Clusters of galaxies are particularly important in this context, because they provide volume-limited samples of galaxies observed at the same cosmic epoch and in an environment corresponding to the highest density peaks in the dark matter distribution at each redshift. It is therefore possible to simultaneously constrain the star formation history (via galaxy colours) and mass assembly history (via the luminosity function) and probe galaxy formation to high redshift, with relatively simple and inexpensive observational campaigns rather than the extensive spectroscopic surveys needed for field galaxies.

Current data show that the colour-magnitude relation of bright galaxies in clusters to $z=1.4$ is very tight and that it evolves passively, thereby implying that their stellar populations must have formed rapidly and at high redshift (e.g. Stanford et al. 1998; Kodama et al. 1998; Papovich et al. 2010 and references therein). Recently, Andreon et al. (2009) detected what seems to be the most distant cluster of galaxies to date (JKCS 041 at $z_{\text {phot }}=2.2$ ) by looking at over densities in red galaxies. In the Chandra follow-up, the cluster is detected as an extended X-ray source with $T \sim 7.4 \mathrm{keV}$ (Andreon et al. 2009; Andreon et al. 2011), which confirms that there is a hot intracluster medium, present in formed clusters and lacking in protoclusters. Deep near-infrared observations show that the cluster has a prominent red sequence (Andreon \& Huertas-Company 2011a,b), locating the cluster at $z_{\text {phot }}=2.20 \pm 0.11$.
Throughout this paper, we assume the following cosmological parameters: $H_{0}=71 \mathrm{~km} \mathrm{~s}^{-1} \mathrm{Mpc}^{-1}, \Omega_{\mathrm{m}}=0.27$, and $\Omega_{\Lambda}=0.73$. Magnitudes are in the AB system.

\section{Data}

JKCS 041 is in the area covered by CFHTLS deep survey and by WIRDS follow-up in the infrared filters $\left(J, K_{\mathrm{s}}\right)$ (catalogues are available on the Terapix site, see also Andreon \& Huertas-Company 2011a,b). More precisely, we used the catalogue generated using $K_{\mathrm{s}}$-band as detection image and the other bands $\left(z^{\prime}, J\right.$ and $\left.H\right)$ in analysis mode. Images have been spatially resampled and filtered during the combining phase, which is why the photometric errors computed by Source Extractor (Bertin \& Arnouts 1996) are underestimated. We assumed an error correction factor of 1.5 for optical images, in agreement with Ilbert et al. (2006) using the very same optical images, and a factor 2.5 for the more finely resampled NIR images, in agreement with the analysis of Raichoor \& Andreon (in prep.) of the very same images.

In this paper, we only consider objects brighter than $K_{\mathrm{s}}=$ 23.0 mag. The claimed $50 \%$ completeness for point sources is 1.9 mag deeper (Bielby et al. 2010). Figure 1 shows the colour-magnitude relation for galaxies within an ellipse of parameters $(\mathrm{ra}, \mathrm{dec}, a, b, P A)=(36.687745,-4.6940845$, $\left.85.6^{\prime \prime}, 55.8^{\prime \prime}, 330\right)$, chosen to maximise the contrast between cluster and background galaxies. In this figure only, blue $\left(z^{\prime}-J<\right.$ $1.75 \mathrm{mag}$ ) galaxies in the SSW octant has been flagged, because this blue part of the diagram is contaminated by a nearby structure (see Andreon \& Huertas-Company 2011a for details). The cluster red sequence clearly stands out. In order to measure the colour spread, and from it the age spread, the colour range to select red galaxies should be large, to avoid very similar colours for the selected galaxies, and as a consequence 


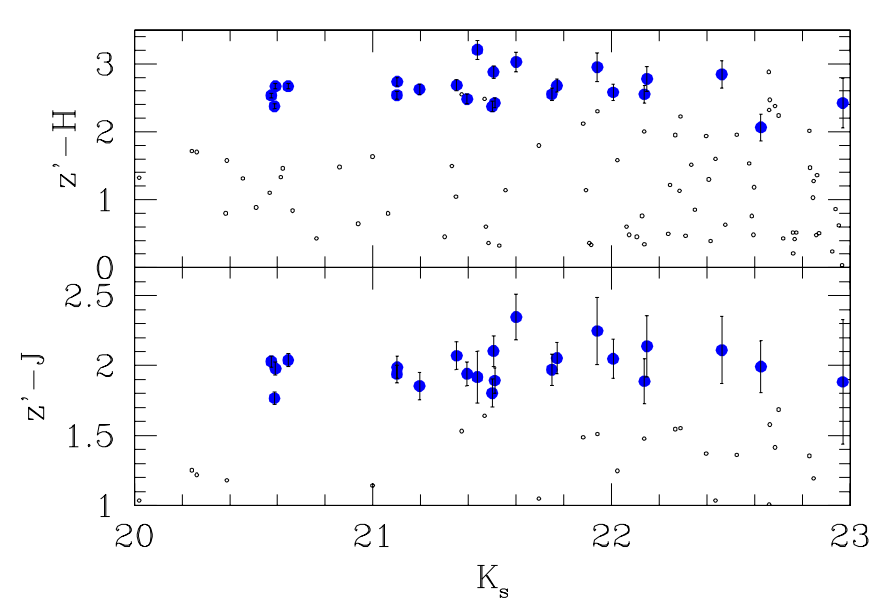

Fig. 1. colour-magnitude plot of galaxies in the JKCS 041 direction. Red sequence $\left(z^{\prime}-J>1.75 \mathrm{mag}\right)$ galaxies are plotted with solid (blue) points. Error bars are marked for these galaxies only, to avoid crowding.

spuriously synchronized star formation histories, because of the too stringent adopted colour cut (see Andreon et al. 2008 for a discussion). We therefore define as red galaxies those with $z^{\prime}-J>1.75 \mathrm{mag}$ and these galaxies are plotted in Fig. 1 as solid points. Bluer galaxies are manifestly too blue to be red sequence galaxies in $z^{\prime}-J$ (they are $>0.22$ away from the red sequence, i.e. $>2.9$ times the red sequence thickness, see also Fig. 2 and Sect. 4). There are 23 galaxies satisfying the constraint above, of which 3.6 are expected to be background galaxies, based on the counts measured in a region of $0.7 \mathrm{deg}^{2}$ all around the cluster. The $z^{\prime}-J$ colour has been adopted because it offers the best way of selecting red cluster galaxies than any other pair of filters with larger wavelength baseline.

Figure 2 shows the colour distribution of the galaxies in the JKCS 041 direction (points with heuristic error bars), and in the background direction, the latter computed from a $0.7 \mathrm{deg}^{2}$ all around the cluster and normalized to the considered cluster solid angle (bottom solid line). In the left panel, all galaxies in the JKCS 041 line of sight are displayed, whereas the $z^{\prime}-J>1.75$ mag selection is applied in the next two panels. These show an isolated peak in the colour distribution, of about 0.18 mag width in $z^{\prime}-H$ and $J-K_{\mathrm{s}}$. The colour distribution of the background galaxies (see bottom solid line) is, as expected, broader and negligible.

\section{Analysis}

The spread across galaxies in star formation history can be inferred from the width of the above colour distributions. The expected spread for a population with an age spread of 50 (250) Myr is plotted in the right (central) panel (dashed blue lines), and the age spread for cluster galaxies should be somewhere in between.

To constrain the star formation history of red galaxies via their colour homogeneity, we were inspired by Bower et al. (1992) and following work, e.g. by Andreon (2003), and Andreon et al. (2009). a) We assumed that the $i$ th galaxy forms stars at the time $t_{i}$, i.e. can be modelled by a single stellar population (SSP, but see later for other choices of star formation histories). b) These individual formation times are spread around the mean star formation age $t$ with a spread $\sigma$ (i.e. $t_{i} \sim \mathcal{N}\left(t, \sigma^{2}\right)$ ).

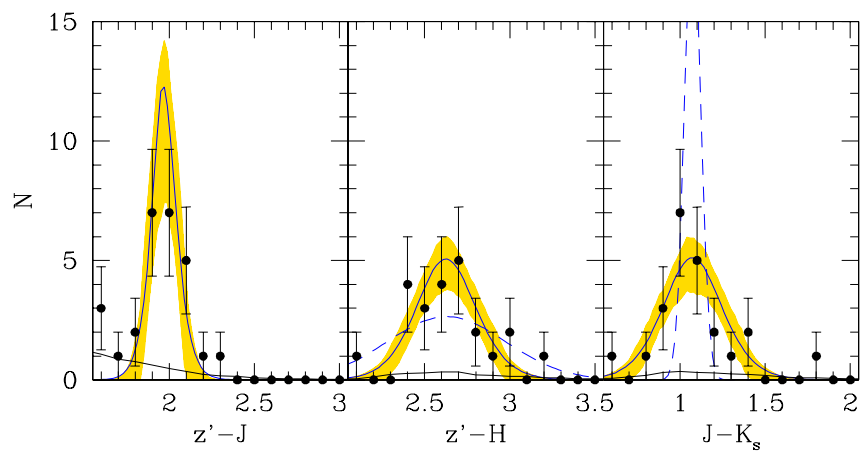

Fig. 2. colour distribution of galaxies in the JKCS 041 direction (points) and in a control region all around the cluster, normalized to the cluster solid angle (lower, black, curve). The (blue) curve with shaded $68 \%$ (highest posterior credible) intervals is a Gaussian fit to the data. Approximated (i.e. $\sqrt{n}$ ) error bars are shown for display purpose only. The dashed blue Gaussian lines show the colour spread expected for a $250 \mathrm{Myr}$ (central panel) or $50 \mathrm{Myr}$ (right panel) age spread.

c) With a stellar population synthesis model, we inferred the colour of each modelled stellar population. d) By a Markov Chain Monte Carlo we updated the parameters $t$ and $\sigma$ until the model colour distribution matched the observed colour distribution. In this step, we account for the heteroscedastic errors, which broaden the colour distribution by an amount that depends on each galaxy. We assumed a uniform prior for $t$, zeroed at $t-\sigma<0$ Gyr (i.e. galaxies form before observing them) and above $t-\sigma>2.54$ Gyr (about $z \sim 8$, only about 0.5 Gyr away from the Big Bang). We assume an uniform prior for the spread $\sigma$, zeroed at $\sigma<0$ because the spread is a positively defined quantity. In our reference analysis, we used the 2007 version of Bruzual \& Charlot (2003) SSP of solar metallicity and Chabrier initial mass function.

As mentioned several times in the literature, the colour evolution is highly sensitive to systematic uncertainties in the stellar population synthesis model (Worthey 1994), and so it is preferable to rely only on differential measurements, i.e. on the colour scatter. We therefore allow stellar population synthesis models to have an unknown zero-point (colour) error as large as \pm 0.4 mag by adding a parameter to the model colour with uniform prior zeroed outside \pm 0.4 mag.

Given the good quality of the data, the posterior is independent of the prior (i.e., results are robust against the prior choice).

\section{Results}

By using $J-K_{\mathrm{s}}$ (rest-frame $U-R$ ) alone, we find a spread of stellar ages of $190 \pm 40 \mathrm{Myr}$. A similar result, $130 \pm 50 \mathrm{Myr}$, is found using $z^{\prime}-H$ alone (rest-frame $U V-V$ ). Using both colours at once, we find a spread of $160 \pm 30 \mathrm{Myr}$. The constraint basically comes from the fact that higher/lower values imply too broad/narrow distributions in colour, independently of the value of the average star formation age $t$. The (posterior) distribution probability of the spread $\sigma$ in star formation age is shown in Fig. 3.

We succeeded at measuring a small scatter in stellar ages because the colour change per Gyr is much higher for younger populations (i.e. at high redshift) than for older ones (i.e. at low redshift). For example, at low redshift, a 0.02 mag colour scatter translates into a 400-700 Myr scatter, depending on the 


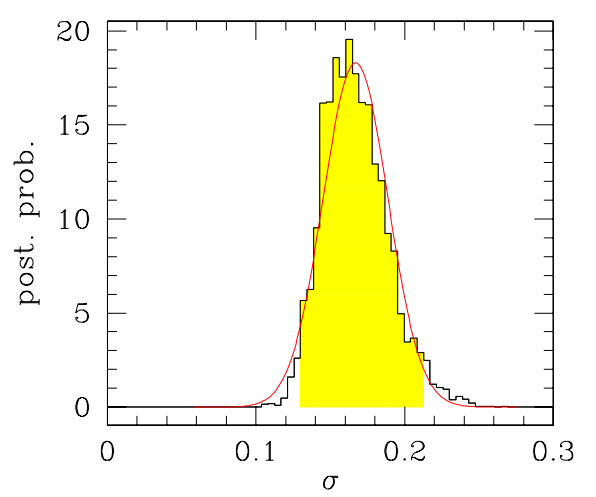

Fig. 3. Posterior probability distribution of the spread $\sigma$ of the stellar age of red JKCS 041 galaxies in Gyr. The shading highlights the $95 \%$ (highest posterior) interval and the red curve is the Gaussian approximation.

adopted plausible age of the stellar population. By going to a high redshift (younger ages), colours change faster, by $0.1 \mathrm{mag}$ per $100 \mathrm{Myr}$, therefore the same 0.02 mag colour scatter corresponds, roughly, to a $20 \mathrm{Myr}$ spread in the star formation age. Furthermore, there is little room for choosing the stellar age, because it is tight bounded by the Big Bang.

To measure the robustness of our result on assumptions, we removed one of them at the time. First, we replaced the SSP model with a model with an exponential decreasing star formation, with $\tau=0.1$ Gyr. We found a spread of $150 \pm 20 \mathrm{Myr}$, less than half a sigma different than adopting SSP's. We had little freedom in choosing much larger $\tau$ models, because those with $\tau>0.5$ have colours that are so blue that they do not match the observed colours, even when allowing for an (implausibly large) model systematic error of $0.4 \mathrm{mag}$. Adopting a $\tau=0.3 \mathrm{Gyr}$, we got a spread of $230 \pm 50 \mathrm{Myr}$, but with tension between the adopted model and the data, because the model is still not red enough to match the data, even when allowing for the 0.4 mag error.

If we use SSP models from Maraston et al. (2005), which use the fuel consumption theorem instead of the isochrones used in Bruzual \& Charlot (2003), we find an identical result: $160 \pm 40$ Myr. If we use a different combination of colour indexes $\left(z^{\prime}-J, J-H, J-K_{\mathrm{s}}\right)$, we find $170 \pm 25 \mathrm{Myr}$, where the error only approximatively accounts for the dependent nature of the three considered colour indexes, again in full agreement with the reference analysis. If we adopt a value of 2.0 for the correction factor for errors in all bands, we find a spread of $180 \pm 30 \mathrm{Myr}$, in full agreement with the reference analysis. If the cluster redshift were $z=2.0$, i.e. two sigmas below the measured value of $z=2.20 \pm 0.11$, then the age spread would be $160 \pm 25 \mathrm{Myr}$, in full agreement with the reference analysis. If we adopt SSP models with twice higher metallicity, then the age-colour relation steepens a bit, and, as a consequence, a narrower spread in ages is needed to reproduce the same observed colour spread. In fact, we find $120 \pm 25 \mathrm{Myr}$, still in line with our reference analysis, since it is only 1.0 combined sigmas away from it.

Our analysis goes from parameters (e.g. age spread) to the data (measured colour spread) in a single step. It may, however, be interesting to take the inverse approach, by inferring the intrinsic scatter of the colour distribution and from it the age scatter. We ignore the background contribution (see Andreon et al. 2008 if one chooses not to ignore it), and we fit the colour and its dispersion accounting for errors with uniform prior on parameters (zeroed for negative, unphysical, dispersions). We find a colour dispersion (red sequence tickness) of $0.18 \pm 0.04$ mag for $z^{\prime}-H$ and $J-K_{\mathrm{s}}$, and $0.08 \pm 0.02 \mathrm{mag}$ in $z^{\prime}-J$. The fits are depicted in Fig. 2. This colour scatter may be inverted, as in Bower et al. (1992) and many works since them, into a 180 Myr age spread. This agrees with our derivation, although we prefer the forward (adopted) approach, instead of inverting the data.

If anything, the measured value is a slightly overestimation of the true age spread, because we have neglected the possibility of a scatter in metallicity or dust content, secondary possibly stochastic minor episodes of star formation superposed on an essentially single stellar population, as well as the (minor) effect of background galaxies among red galaxies and the negligible effect of the colour-magnitude slope. All these terms tend to increase the expected observational scatter at a given age scatter $\sigma$, unless there is a tuned covariance between them to keep the observed colour scatter small.

\section{Conclusions}

We found an age spread of $160 \pm 30 \mathrm{Myr}$, independently of the assumptions used to infer ages from a colour scatter, namely the absolute colour of the stellar populations, the synthesis population code used, the way the star formation history of each galaxy is modelled, the adopted metallicity, the choice of the colour index, and the precise redshift of the cluster and the approach (forward or inverse) used in analysing the data. We succeeded at measuring such a small scatter in stellar ages because the colour change per Gyr is much higher for younger populations (i.e. at high redshift) than for older ones (i.e. at low redshift), and because the range of possible ages is much reduced at high redshift, as we are approaching the Big Bang.

The extreme synchronicity of red galaxies in JKCS 041 is in marked disagreement with the idea that galaxies in clusters form by a hierarchical assembly of smaller units, unless this occurs a) over such a short time that it is essentially equivalent to an (un-physical) monolithic assembly; or b) by gas-free merging. In fact, a hierarchical assembly has some stochastic variations in the merging histories, which induce differences in colours because some merging events involve gas. It is worth noticing that the Menci et al. (2008) rendition of the hierarchical paradigm predicts the disappearance of the red sequence in clusters at redshift around 1.5 to 2.0 .

In the $z=2.2 \mathrm{JKCS} 041$ cluster, the red sequence is already populated by a homogenous population of galaxies with extremely synchronised stellar ages, which implies that mechanisms not yet implemented in current galaxy formation scenarios long ago began to shape the star formation histories of red cluster galaxies.

Acknowledgements. I thank Anand Raichoor for useful discussions and Claudia Maraston for her models in electronic form. This work is based on observations with MegaPrime/MegaCam ${ }^{1}$ and WIRCAM ${ }^{2}$ at CFHT.

\footnotetext{
${ }^{1}$ The full text acknowledgement is at http://www.cfht.hawaii. edu/Science/CFHLS/cfhtlspublitext.html

2 The full text acknowledgement is at http://ftp.cfht.hawaii. edu/Instruments/Imaging/WIRCam/WIRCamAcknowledgment . html
} 
A\&A 529, L5 (2011)

\section{References}

Andreon, S. 2001, ApJ, 547, 623

Andreon, S. 2003, A\&A, 409, 37

Andreon, S. 2006a, A\&A, 448, 447

Andreon, S. 2006b, MNRAS, 369, 969

Andreon, S. 2008, MNRAS, 386, 1045

Andreon, S., \& Huertas-Company, M. 2011a, A\&A, 526, A11

Andreon, S., \& Huertas-Company, M. 2011b, Mem. Soc. Astron. Ital., in press [arXiv: $1012.3051 \mathrm{~A}$ ]

Andreon, S., Cuillandre, J.-C., Puddu, E., \& Mellier, Y. 2006, MNRAS, 372, 60 Andreon, S., Puddu, E., de Propris, R., \& Cuillandre, J.-C. 2008, MNRAS, 385, 979

Andreon, S., Maughan, B., Trinchieri, G., \& Kurk, J. 2009, A\&A, 507, 147
Andreon, S., Trinchieri, G., \& Pizzolato, F. 2011, MNRAS, in press [arXiv: 1012.3034]

Bertin, E., \& Arnouts, S. 1996, A\&AS, 117, 393

Bielby, R. M., Finoguenov, A., Tanaka, M., et al. 2010, A\&A, 523, A66

Bower, R. G., Lucey, J. R., \& Ellis, R. S. 1992, MNRAS, 254, 601

Bruzual, G., \& Charlot, S. 2003, MNRAS, 344, 1000

Ilbert, O., Arnouts, S., McCracken, H. J., et al. 2006, A\&A, 457, 841

Kodama, T., Arimoto, N., Barger, A. J., Arag'on-Salamanca, A. 1998, A\&A, 334, 99

Maraston, C. 2005, MNRAS, 362, 799

Menci, N., Rosati, P., Gobat, R., et al. 2008, ApJ, 685, 863

Papovich, C., Momcheva, I., Willmer, C. N. A., et al. 2010, ApJ, 716, 1503

Stanford, S. A., Eisenhardt, P. R., \& Dickinson, M. 1998, ApJ, 492, 461

Worthey, G. 1994, ApJS, 95, 107 